\title{
Effects of $\beta_{6}$ deformation and low-lying vibrational bands on heavy-ion fusion reactions at sub-barrier energies
}

\author{
Tamanna Rumin ${ }^{1},{ }^{*}$ Kouichi Hagino ${ }^{2},{ }^{\dagger}$ and Noboru Takigawa ${ }^{1} \ddagger$ \\ ${ }^{1}$ Department of Physics, Tohoku University, Sendai 980-8578, Japan \\ 2 Institute for Nuclear Theory, Department of Physics, University of Washington, Seattle, WA 98195
}

(August 2, 2018)

\begin{abstract}
We study fusion reactions of ${ }^{16} \mathrm{O}$ with ${ }^{154} \mathrm{Sm},{ }^{186} \mathrm{~W}$ and ${ }^{238} \mathrm{U}$ at sub-barrier energies by a coupledchannels framework. We focus especially on the effects of $\beta_{6}$ deformation and low-lying vibrational excitations of the target nucleus. It is shown that the inclusion of $\beta_{6}$ deformation leads to a considerable improvement of the fit to the experimental data for all of these reactions. For the ${ }^{154} \mathrm{Sm}$ and ${ }^{238} \mathrm{U}$ targets, the octupole vibration significantly affects the fusion barrier distribution. The effect of $\beta$ band is negligible in all the three reactions, while the $\gamma$ band causes a non-negligible effect on the barrier distribution at energies above the main fusion barrier. We compare the optimum values of the deformation parameters obtained by fitting the fusion data with those obtained from inelastic scatterings and the ground state mass calculations. We show that the channel coupling of high multipolarity beyond the quadrupole coupling is dominated by the nuclear coupling and hence higher order Coulomb coupling does not much influence the optimum values of $\beta_{4}$ and $\beta_{6}$ parameters. We also discuss the effect of two neutron transfer reactions on the fusion of ${ }^{16} \mathrm{O}$ with ${ }^{238} \mathrm{U}$.
\end{abstract}

25.70.Jj, 21.60.Ev, 24.10.Eq, 23.20.Js

\section{INTRODUCTION}

It is now well established that nuclear intrinsic motions significantly enhance the fusion cross-section in heavy-ion reactions at sub-barrier energies. Deformation effect is one of such prominent effects. The role of static deformation in enhancing fusion cross section has long been recognized [1.2] and has been experimentally demonstrated [3 5]. Here the enhancement occurs because there is a distribution of barrier heights which can be thought of as resulting from different orientations of the deformed target nucleus. Any distribution of barriers around a single Coulomb barrier leads to enhancement of the fusion cross section at energies below the single barrier, because passes through the lower barriers are much more probable. Recently, high precision experimental data were obtained for the ${ }^{16} \mathrm{O}+{ }^{186} \mathrm{~W},{ }^{154} \mathrm{Sm}$ fusion reactions and it was clearly demonstrated that sub-barrier fusion reactions strongly depend upon the nuclear hexadecapole deformation [6.,7. It was pointed out that the optimum values of the quadrupole and hexadecapole deformation parameters obtained from the analyses of such high precision fusion data are consistent with those obtained from the Coulomb excitation [8,9] using similar radius parameter [10]. In order to reach this conclusion, the authors of Refs. 66,0,10] included up to the $\beta_{4}$ deformation in their analyses, neglecting higher order deformations such as $\beta_{6}$. On the other hand, the differential cross sections of inelastic alpha particle [11] and proton [12] scatterings from ${ }^{154} \mathrm{Sm}$ and neutron scattering from ${ }^{186} \mathrm{~W}$ [13 show important effects of $\beta_{6}$ deformation of the target nuclei. The important role of $\beta_{6}$ deformation of the target nucleus has been shown also in the inelastic alpha and proton scatterings from ${ }^{238} \mathrm{U}$, which give optimum deformation parameters consistent to each other [14 17. Although each reaction might be sensitive to different channels, it is not obvious whether the effects of $\beta_{6}$ deformation on fusion reactions are negligible. We also notice that there still remain noticeable discrepancies between the experimental and theoretical barrier distributions in Ref. [7] which included up to $\beta_{4}$ deformation. Although a better reproduction of the experimental data of the fusion excitation function as well as the fusion barrier distribution has been obtained by including the effects of low-lying two $2^{+}$and one $3^{-}$vibrations and a positive Q-value transfer channel, the role of higher order deformations has not yet been clarified. This motivated the present work, where we perform a detailed study of the effects of higher order deformation, especially the effects of $\beta_{6}$ deformation on heavy-ion fusion reactions.

\footnotetext{
*E-mail address: rumin@nucl.phys.tohoku.ac.jp

${ }^{\dagger}$ E-mail address: hagino@phys.washington.edu

${ }^{\ddagger}$ E-mail address: takigawa@nucl.phys.tohoku.ac.jp
} 
Besides clarifying the mechanism of heavy-ion fusion reactions, the study of the effects of higher order deformation is interesting to see the possibility of heavy-ion fusion reactions as a new powerful method of nuclear spectroscopy. This is another motivation of the present work. We therefore compare the optimum values of the deformation parameters obtained from the analyses of the fusion data with those from inelastic scatterings and the ground state mass calculations. In order to have reliable results, one has to take various channel coupling mechanisms into account which might cause effects of similar order. In this connection, we discuss in this paper the effects of vibrational excitations of deformed targets. We also pay attention to the role of higher order Coulomb coupling.

For these purposes, we particularly study ${ }^{16} \mathrm{O}+{ }^{154} \mathrm{Sm},{ }^{186} \mathrm{~W},{ }^{238} \mathrm{U}$ fusion reactions, where high precision experimental data have been obtained [7, 18]. We discuss the effects of channel coupling through the excitation function of the fusion cross section and the fusion barrier distribution [19], which is defined as the second derivative of the product of the bombarding energy $E$ and the fusion cross section $\sigma_{F}$ with respect to $E$. Though the fusion barrier distribution has, strictly speaking, clear physical meaning only in the limit of sudden fusion, i.e. in the limit where the excitation energy of intrinsic excitations can be ignored, it has been shown that the concept still holds to a good approximation even for non-zero excitation energy [20]. This method has often been used to analyse high precision heavy-ion fusion data and is now well known to provide a very sensitive test of various channel coupling effects.

The paper is organized as follows. In Sec. II, we present the results of the coupled-channels analysis which takes only the ground state rotational band into account. The main result is that $\beta_{6}$ deformation plays an important role in all the three reactions. For ${ }^{186} \mathrm{~W}$, the magnitude as well as the sign of the $\beta_{6}$ are consistent with the results of ground state mass calculations and inelastic neutron scattering. However, the sign of $\beta_{6}$ for the $\mathrm{Sm}$ and $\mathrm{U}$ targets is predicted to be opposite to the result of other studies. In Sec. III, we examine the validity of the calculations used in Sec. II, which take full order for the nuclear coupling, while only the linear order for the Coulomb coupling into account. By performing coupled-channels calculations keeping up to the second order in the Coulomb coupling, we show that the high multipolarity couplings, i.e. the $\mathrm{Y}_{4}$ and $\mathrm{Y}_{6}$ couplings, are dominated by the nuclear coupling and hence non-linear Coulomb coupling does not almost alter the optimum values of $\beta_{4}$ and $\beta_{6}$. In Sec.IV we present the results of coupled-channels analyses for ${ }^{16} \mathrm{O}+{ }^{154} \mathrm{Sm}$ and ${ }^{238} \mathrm{U}$ fusion reactions which take octupole vibrations into account. These target nuclei have low-lying $\mathrm{K}=0^{-}$octupole bands, which are strongly excited by the Coulomb excitation. The E3 transition strength from the ground state to the $3^{-}$state is 24 W.u. for ${ }^{238} \mathrm{U}$ and $11 \mathrm{~W} . \mathrm{u}$. for ${ }^{154} \mathrm{Sm}$. We show that the octupole vibration significantly affects the fusion barrier distribution and modifies the optimum values of deformation parameters to fit the experimental data. Especially, it changes the sign of $\beta_{6}$ deformation to agree with the analyses of inelastic $\alpha$ and proton scatterings and the ground state mass calculations. Notice that there is no experimental evidence for the low-lying octupole $\mathrm{K}=0^{-}$band in ${ }^{186} \mathrm{~W}$, suggesting its absence in this nucleus. All the three target nuclei have low-lying $\beta$ and $\gamma$ bands, whose interband E2 transition probabilities from the ground state $0^{+}$to the $2^{+}$member are: 1.0 and 4.4 W.u. in ${ }^{154} \mathrm{Sm}, 8.9$ and 1.4 W.u. in ${ }^{186} \mathrm{~W}$ and 3.0 and 1.5 W.u. in ${ }^{238} \mathrm{U}$ for the $\gamma$ and $\beta$ bands, respectively. In Sec.V, we examine the effects of $\beta$ and $\gamma$ vibrations, and show that the effect of $\beta$ band is negligible, while the $\gamma$ band affects the fusion barrier distribution at high energies. Besides nuclear intrinsic excitations, nucleon transfer reactions between the colliding nuclei can enhance the low-energy fusion cross section. In Sec.VI, we study the effect of pair neutron transfer reactions on the ${ }^{16} \mathrm{O}+{ }^{238} \mathrm{U}$ fusion reactions and discuss whether it explains the experimental fusion cross section which is systematically larger than the prediction of the coupled-channels calculations which ignore particle transfer reactions at low energies. We summarize the paper in Sec.VII. Appendix A is added to briefly explain the theoretical framework of the coupled-channels calculations we use. We also add Appendix B to show the structure of the higher order Coulomb coupling.

\section{EFFECT OF $\beta_{6}$ DEFORMATION}

In this section we present the results of coupled-channels calculations which take only the ground state rotational band of the target nucleus into account. We treat the projectile as inert, since its excitations can be well incorporated with a choice of the bare potential [21]. Instead of handling the full coupled-channels equations, we introduce the no-Coriolis approximation throughout this paper, and ignore the change of the centrifugal potential barrier due to the finite multipolarity of nuclear intrinsic excitations [22,23]. This leads to considerable reduction of the dimension of the coupled-channels equations. We assume an axially symmetric deformation for the target nucleus and expand the radius up to the hexacontatetrapole deformation $\beta_{6}$. We introduce the sudden tunneling approximation, and set the excitation energy of the ground state $\mathrm{K}=0^{+}$rotational band to zero. Together with the no-Coriolis approximation, this leads to a set of decoupled eigen-channel problems, each of which corresponds to the fusion with a fixed orientation of the target nucleus. Accordingly, we first solve the Schrödinger equation for a given orientation $\theta$ for each partial wave $J$ using the incoming wave boundary condition to obtain the tunneling probability $P_{J}(E, \theta)$. We then calculate the total tunneling probability $P_{J}(E)$ for each $J$ by taking average over all orientations as, 


$$
P_{J}(E)=\frac{1}{2} \int_{0}^{\pi} P_{J}(E, \theta) \sin \theta d \theta
$$

where the weight of the average has been determined by the ground state wave function of the deformed target, which is initially in the $0^{+}$state. The fusion cross section is then obtained by the standard partial wave sum. Once the fusion excitation function has been obtained, the fusion barrier distribution is calculated by the point difference formula of $\Delta E=2 \mathrm{MeV}$ in the laboratory energy, whose value was employed in Refs. [6., 7.10] in analyzing the experimental data.

We first determine the nuclear potential parameters for each target nucleus by fitting the fusion cross section larger than $200 \mathrm{mb}$ by a potential model [7]. We then calculate the fusion cross section by switching on deformations of different multipolarity successively. At each step, we determine the values of the deformation parameters by $\chi^{2}$ fitting of the data of fusion excitation function and readjust the potential parameters. We use the values in Ref. [7] as the initial values. The results are shown in Fig.1. The left and right columns are the excitation function of the fusion cross section and the fusion barrier distribution, respectively. The top, center and bottom panels are for ${ }^{154} \mathrm{Sm},{ }^{186} \mathrm{~W}$ and ${ }^{238} \mathrm{U}$ targets, respectively. The dashed, the dotted and the solid lines represent the results of coupled-channels calculations including only $\beta_{2}$ deformation, $\beta_{4}$ in addition and $\beta_{6}$ as well, respectively. The dashed line for the ${ }^{16} \mathrm{O}+{ }^{186} \mathrm{~W}$ fusion reactions cannot be seen clearly in the fusion excitation function, because it overlaps with the solid line in the semi-logarithmic plot of the present scale. However, it is clearly separated from the other two lines in the fusion barrier distribution. This typically shows the high sensitivity of the fusion barrier distribution to different channel coupling effects. The deformation parameters obtained in the analysis are shown in the figure. Those obtained including only up to $\beta_{4}$ deformation for the ${ }^{154} \mathrm{Sm}$ and ${ }^{186} \mathrm{~W}$ targets somewhat differ from those obtained in [7], which are $\beta_{2}=0.33, \beta_{4}=0.05$ and $\beta_{2}=0.31, \beta_{4}=-0.03$, respectively. Since we use the same radius parameter as in Ref. [7], these differences can probably be attributed to the different methods to calculate the fusion cross section in two works. We calculated it by numerically solving the Schrödinger equations, while Ref. [7] introduced the parabolic barrier approximation, which does not work at energies far below the barrier.

The importance of the $\beta_{6}$ deformation can be clearly seen in the fusion barrier distribution for all cases, and in the fusion excitation function as well for the ${ }^{154} \mathrm{Sm}$ and ${ }^{238} \mathrm{U}$ targets. The agreement between the experimental data and the coupled-channels calculations concerning the fusion barrier distribution has been significantly improved above 56 and $66 \mathrm{MeV}$ for ${ }^{154} \mathrm{Sm}$ and ${ }^{186} \mathrm{~W}$, respectively, by including $\beta_{6}$ deformation. The $\beta_{6}$ deformation removes a sharp peak at around $82 \mathrm{MeV}$ in the fusion barrier distribution, which appears in the coupled-channels calculations without $\beta_{6}$ deformation, for the ${ }^{238} \mathrm{U}$ target.

In Fig.2, we show the dependence of the fusion excitation function and the fusion barrier distribution on the $\beta_{6}$ parameter for the ${ }^{16} \mathrm{O}+{ }^{154} \mathrm{Sm},{ }^{186} \mathrm{~W}$ reactions. The three lines in each figure have been calculated by using the same parameter sets as in Fig.1 (the solid lines), or by inverting the sign of the $\beta_{6}$ parameter (the dotted lines) and by setting it to be zero (the dashed lines). This figure also shows that the effect of $\beta_{6}$ deformation on the fusion cross section is not negligible.

We compare in Table 1 the optimum values of the deformation parameters thus obtained with those obtained from the analyses of inelastic scatterings and the ground state mass calculations. The table also shows the radius parameter used in each analysis. For all three target nuclei, we observe noticeable discrepancies in the magnitudes of the deformation parameters among different studies (magnitude problem). This problem is, however, not so serious as it appears, because the discrepancies are largely due to the different choice of the radius parameter in each analysis. Since the strength of the channel coupling depends on the product of the deformation and radius parameters for the nuclear part and on $\beta_{\lambda} \times R_{T}^{\lambda}$ for the Coulomb part (see Eqs.(A.2-A.4)), physically important quantities are these products. Based on this idea, Table 1 shows the scaled deformation parameters as well, which have been calculated by $\beta_{\lambda} \times r_{0} / 1.06$ (figures with a star) or by $\beta_{\lambda} \times\left(r_{c} / 1.06\right)^{\lambda}$ (figures with two stars) from the original deformation parameters. We observe that the scaled deformation parameters from non-fusion studies are now much closer to the optimum deformation parameters from the fusion analysis. We cannot unfortunately rescale the deformation parameters for the neutron scattering from ${ }^{186} \mathrm{~W}$, since the radius parameter is not given in [13].

We wish to especially remark that the sign and the magnitude of $\beta_{6}$ obtained from fusion analysis are consistent with those obtained from the ground state mass calculations for the ${ }^{186} \mathrm{~W}$ target. Our result is consistent also with the neutron scattering, though it gives only the upper bound of the magnitude. On the other hand, the predicted sign of $\beta_{6}$ is opposite to the results of other studies for ${ }^{154} \mathrm{Sm}$ and ${ }^{238} \mathrm{U}$ (sign problem). We show in Sec. IV that the effect of octupole vibration provides a possibility to cure this sign problem. We note that the optimum deformation parameters of ${ }^{154} \mathrm{Sm}$ and ${ }^{238} \mathrm{U}$ obtained from many experiments of proton and $\alpha$ particle scatterings agree quite well to each other including the sign and magnitude of $\beta_{6}$, though there exist a few exceptions in the case of ${ }^{154} \mathrm{Sm}$.

For reference, we compare in Fig.3 the experimental data and the results of coupled-channels calculations using deformation parameters from the fusion data (the solid line), the ground state mass calculations [24] (the dotted line) and inelastic scatterings (the dashed line). For the latter, we choose the results of inelastic alpha [11], neutron [13] and proton [17] scatterings for ${ }^{154} \mathrm{Sm},{ }^{186} \mathrm{~W}$ and ${ }^{238} \mathrm{U}$, respectively. In calculating the dotted and the dashed lines, 
the radius of the target nucleus $R_{T}$ has been adjusted such that $\beta_{2} R_{T}$ equals that in the fusion calculations, where we chose $R_{T}=1.06 \times \mathrm{A}_{T}^{1 / 3} \mathrm{fm}$ in order to have the same nuclear quadrupole coupling as for the solid line. Naturally, the parameter set obtained by the $\chi^{2}$ fit of the fusion data provides the best fit to the experimental data.

Table 1: Comparison of the optimum deformation parameters and nuclear radius parameter in various analyses.

\begin{tabular}{|c|c|c|c|c|c|c|}
\hline Nuclei & Methods & $r_{0}(\mathrm{fm})$ & $r_{c}(\mathrm{fm})$ & $\beta_{2}$ & $\beta_{4}$ & $\beta_{6}$ \\
\hline \multirow[t]{6}{*}{${ }^{154} \mathrm{Sm}$} & ${ }^{16} \mathrm{O}+{ }^{154} \mathrm{Sm}$ Fusion & 1.06 & $\begin{array}{l}\ldots \\
\end{array}$ & 0.322 & 0.027 & 0.027 \\
\hline & Mass Calculation 24] & 1.16 & $\cdots$ & 0.27 & 0.113 & -0.005 \\
\hline & & & & $0.295 *$ & $0.124 *$ & $-0.005 *$ \\
\hline & $\alpha$ Scattering [1] & 1.492 & & $0.225 \pm 0.005$ & $0.050 \pm 0.005$ & $-0.015 \pm 0.010$ \\
\hline & & & & $0.317 *$ & $0.070 *$ & $-0.021 *$ \\
\hline & proton Scattering [12] & & $\ldots$ & 0.285 & 0.051 & -1 \\
\hline \multirow[t]{4}{*}{${ }^{186} \mathrm{~W}$} & ${ }^{16} \mathrm{O}+{ }^{186} \mathrm{~W}$ Fusion & 1.06 & $\cdots$ & 0.285 & -0.031 & 0.027 \\
\hline & Mass Calculation [24] & 1.16 & $\cdots$ & 0.23 & -0.107 & 0.02 \\
\hline & & & & $0.25 *$ & $-0.117 *$ & $0.022 *$ \\
\hline & neutron Scattering [13] & $\ldots$ & $\ldots$ & $0.203 \pm 0.006$ & $-0.057 \pm 0.006$ & $|<|-0.04 \mid$ \\
\hline \multirow[t]{7}{*}{${ }^{238} \mathrm{U}$} & ${ }^{16} \mathrm{O}+{ }^{238} \mathrm{U}$ Fusion & 1.06 & $\cdots$ & 0.289 & 0.01 & 0.044 \\
\hline & Mass Calculation [24] & 1.16 & $\ldots$ & 0.215 & 0.093 & -0.015 \\
\hline & 吸 & & & $0.235 *$ & $0.102 *$ & $-0.016 *$ \\
\hline & $\alpha$ Scattering [14] & 1.2 & .... & $0.22 \pm 0.01$ & $0.06 \pm 0.01$ & $-0.012 \pm 0.01$ \\
\hline & & & & $0.25 *$ & $0.068 *$ & $-0.014 *$ \\
\hline & proton Scattering [17] & $\cdots$ & 1.25 & $0.225 \pm 0.005$ & $0.045 \pm 0.005$ & $-0.015 \pm 0.003$ \\
\hline & & & & 0.31 & 0.087 & -0.040 \\
\hline
\end{tabular}

\section{HIGHER ORDER COULOMB COUPLING}

The results in Sec. II have been obtained by treating the Coulomb coupling in the linear order and the nuclear coupling in full order. Though this approximation is often used in literatures, it is worth checking the validity, especially in discussing the role of higher order deformations. One would guess that this approximation breaks down when the charge product of the projectile and target gets large. As examples, we performed coupled-channels calculations for the ${ }^{32} \mathrm{~S}+{ }^{168} \mathrm{Er}$ and ${ }^{16} \mathrm{O}+{ }^{154} \mathrm{Sm}$ fusion reactions by including the second order Coulomb coupling and by assuming only the quadrupole coupling. We found that the second order Coulomb coupling noticeably modifies the fusion barrier distribution. Naturally, the modification is more significant for the former reaction. An important issue in the context of the present paper is whether the higher order Coulomb coupling significantly changes the optimum values of higher order deformation parameters that reproduce the experimental data of fusion cross section. In this connection, we show in Appendix B the higher order terms in the Coulomb interaction up to the order of $\beta_{6}$, i.e. $\beta_{4} \times \beta_{2}$. Although $\beta_{2}^{3}$ would contribute in the same order, we do not show it, since it is very tedious to evaluate it and also its effects are negligible as we argue below. Note that other terms, like $\beta_{4}^{2}$ and $\beta_{2} \times \beta_{6}$, are higher order contributions, which are the same order of $\beta_{8}$ or higher, and are not shown. Eq.(B.1) indicates that the optimum values of $\beta_{4}$ and $\beta_{6}$ parameters will be considerably altered by the non-linear coupling if the Coulomb coupling significantly contributes to the higher multipolarity, i.e. $\mathrm{Y}_{4}$ and $\mathrm{Y}_{6}$, couplings.

In order to examine the situation, we compare in Fig.4 the fusion barrier distribution calculated in four different ways. For simplicity, all the calculations have been performed by treating both the nuclear and Coulomb couplings in linear order and by expanding up to the $\mathrm{Y}_{6}$ term. The solid line is the fusion barrier distribution obtained by keeping both the nuclear and Coulomb couplings as they are. The dashed line has been obtained by discarding the nuclear $\mathrm{Y}_{4}$ coupling term. We observe a significant change of the fusion barrier distribution. We performed additional two calculations, where only the Coulomb or both the nuclear and Coulomb $\mathrm{Y}_{4}$ couplings are discarded. Their results are almost the same as the solid and the dashed lines, respectively. These results indicate that the $\mathrm{Y}_{4}$ coupling is far dominated by the nuclear coupling. We checked that a similar situation holds also for the $\mathrm{Y}_{6}$ coupling. We thus conjecture that the main effect of the Coulomb coupling resides in the $\mathrm{Y}_{2}$ coupling, and one can determine to a good approximation the optimum $\beta_{4}$ and $\beta_{6}$ parameters through the coupled-channels calculations using the linear Coulomb coupling. In the following analyses, we thus treat the Coulomb coupling in the linear order. Keep, however, in mind that the optimum $\beta_{2}$ value could be noticeably affected depending on whether one uses the linear or higher order Coulomb coupling. 


\section{EFFECT OF OCTUPOLE VIBRATION}

We now study the effects of octupole vibration on the ${ }^{16} \mathrm{O}+{ }^{154} \mathrm{Sm}$ and ${ }^{16} \mathrm{O}+{ }^{238} \mathrm{U}$ fusion reactions. As already mentioned in the introduction, there exist low-lying $\mathrm{K}=0^{-}$octupole bands in ${ }^{154} \mathrm{Sm}$ and ${ }^{238} \mathrm{U}$, which are strongly excited by the Coulomb excitation through the E3 transition. We take into account their effects on fusion by solving coupled-channels equations for each orientation of the deformed target. We call this procedure the $\theta$-scheme. We confirmed that the results are almost the same as those obtained by treating the rotational excitations not by the $\theta$-scheme, but by specifying each excited level by its spin, and by solving coupled-channels equations with a larger dimension which include both the $\mathrm{K}=0^{+}$ground state and $\mathrm{K}=0^{-}$octupole bands [25]. Similarly to the rotational coupling, we treat the nuclear part of the octupole coupling in full order, while the Coulomb part in the linear order. The amplitude of the zero point motion of the octupole vibration, which governs the strength of the channel-coupling, is determined from the experimental value of the reduced transition probability $\mathrm{B}\left(\mathrm{E} 3_{o} \uparrow\right)$ [26, 27] from the ground state to the $3^{-}$state of the $\mathrm{K}=0^{-}$octupole vibrational band following

$$
\alpha_{0}^{o}=\frac{\left(\frac{4 \pi}{3 Z R_{T}^{3}}\right) \sqrt{B\left(E 3_{o} \uparrow\right) / e^{2}}}{\left[1+\frac{1}{3} \sqrt{\frac{5}{\pi}} \beta_{2}+\frac{5}{22} \sqrt{\frac{9}{\pi}} \beta_{4}+\frac{125}{13 \times 11 \times 3} \sqrt{\frac{13}{\pi}} \beta_{6}\right]} .
$$

The optimum set of deformation parameters as well as the potential parameters are readjusted by the $\chi^{2}$ fitting after including the $3^{-}$vibrational state. The results are shown in Fig.5 by solid lines in comparison with the experimental data and the previous calculations which include only the ground state rotational band (the dashed lines). The effects of the octupole vibration are visible especially in the fusion barrier distribution. The resultant optimum deformation parameters are $\beta_{2}=0.314, \beta_{4}=0.011$ and $\beta_{6}=-0.016$ for ${ }^{154} \mathrm{Sm}$ and $\beta_{2}=0.279, \beta_{4}=0.0007$ and $\beta_{6}=-0.024$ for ${ }^{238} \mathrm{U}$. An interesting result is that the sign problem of $\beta_{6}$ parameter has been resolved for both ${ }^{154} \mathrm{Sm}$ and ${ }^{238} \mathrm{U}$ nuclei, although the optimum values of $\beta_{4}$ become too small, especially in ${ }^{238} \mathrm{U}$, compared with the other analyses.

\section{SIMULTANEOUS EFFECTS OF OCTUPOLE, $\beta$ AND $\gamma$ VIBRATIONS}

We now add the effects of the $\beta$ and $\gamma$ vibrations. We treat all the vibrational excitations by a coupled-channels framework by keeping their finite excitation energies and using the linear coupling approximation not only for the Coulomb but also for the nuclear parts. The rotational coupling is treated in the same way as in Sec. II.

The amplitudes of the zero point motion of the $\beta$ and $\gamma$ vibrations are determined from the experimental values of the reduced transition probability $\mathrm{B}(\mathrm{E} 2 \uparrow)$ [28] from the ground state to the $2^{+}$state of the $\beta$ band and to the band head of the $\gamma$ band. The formulae we use are,

$$
\alpha_{0}{ }^{\beta}=\frac{\sqrt{B\left(E 2_{\beta} \uparrow\right) / e^{2}}}{\left(\frac{3 Z R_{T}^{2}}{4 \pi}\right)\left(1+\frac{4}{7} \sqrt{\frac{5}{\pi}} \beta_{2}\right)}, \quad \alpha_{0}^{\gamma}=\frac{\sqrt{B\left(E 2_{\gamma} \uparrow\right) / 2 e^{2}}}{\left(\frac{3 Z R_{T}^{2}}{4 \pi}\right)\left(1-\frac{4}{7} \sqrt{\frac{5}{\pi}} \beta_{2}\right)} .
$$

Table 2 collects the experimental transition probabilities and the values of the zero point motion amplitudes for the $\beta$ and $\gamma$ vibrations together with those for the octupole vibration. It includes also $\beta_{2}$ values. They have been extracted in Sec. II and used to determine $\alpha_{0}^{\beta}, \alpha_{0}^{\gamma}$ and $\alpha_{0}^{o}$ following Eqs. 3 and 2 .

\begin{tabular}{|c|c|c|c|c|c|c|c|}
\hline Nuclei & $B\left(E 3_{o} \uparrow\right)\left(e^{2} b^{3}\right)$ & $B\left(E 2_{\beta} \uparrow\right)\left(e^{2} b^{2}\right)$ & $B\left(E 2_{\gamma} \uparrow\right)\left(e^{2} b^{2}\right)$ & $\beta_{2}$ & $\alpha_{0}^{o}$ & $\alpha_{0}^{\beta}$ & $\alpha_{0}^{\gamma}$ \\
\hline${ }^{154} \mathrm{Sm}$ & 0.100 [26] & 0.023 [28 & \begin{tabular}{|l|l|}
0.069 & 28 \\
\end{tabular} & 0.322 & 0.103 & 0.026 & 0.051 \\
\hline${ }^{186} \mathrm{~W}$ & |... & 0.009 & 0.150 & 0.285 & $\ldots$ & 0.012 & 0.054 \\
\hline${ }^{238} \mathrm{U}$ & 0.575 27] & 0.0656 [28] & 0.131 [28] & 0.289 & 0.109 & 0.0224 & 0.034 \\
\hline
\end{tabular}

Table 2 : The zero point motion amplitude of the octupole, $\beta$ and $\gamma$ vibrations.

Since the coupling to the $\gamma$ band depends on the second Euler angle $\phi$, we first solve the coupled-channels equations for a given set of $(\theta, \phi)$ parameters. The fusion cross section for each partial wave $J$ is then calculated by taking average over both $\theta$ and $\phi$,

$$
P_{J}(E)=\frac{1}{4 \pi} \int_{0}^{\pi} \sin \theta d \theta \int_{0}^{2 \pi} d \phi P_{J}(E, \theta, \phi)
$$


The integrations are performed by Gauss quadrature. Since the numerical computation is quite heavy, we have not optimized the deformation parameters, but fixed them to those values used to obtain the solid line in Fig. 1 for ${ }^{16} \mathrm{O}+{ }^{186} \mathrm{~W}$ and those in Fig.5 for ${ }^{16} \mathrm{O}+{ }^{154} \mathrm{Sm}$ and ${ }^{16} \mathrm{O}+{ }^{238} \mathrm{U}$ fusion reactions.

The results are shown in Fig.6. Similarly to Fig.1, the fusion excitation function and the fusion barrier distribution are shown on the left and right sides, respectively. The solid line for the ${ }^{16} \mathrm{O}+{ }^{186} \mathrm{~W}$ fusion reaction was obtained by including the effect of only the ground state rotational band, and is the same as the solid line in Fig.1, while the solid line for the ${ }^{16} \mathrm{O}+{ }^{154} \mathrm{Sm}$ and ${ }^{16} \mathrm{O}+{ }^{238} \mathrm{U}$ fusion reactions was obtained by adding the octupole vibration. It is slightly different from the solid line in Fig. 5 because of the different treatment of the nuclear coupling.

The effect of the $\beta$ band is very small for all three systems and invisible in the scale of Fig.6. The dotted line was obtained by adding the effect of $\gamma$ band. Its effect is less important than that caused by the $\beta_{4}$ and $\beta_{6}$ deformations, but is noticeable in the fusion barrier distribution. An interesting thing is that the $\gamma$ band does not affect the fusion excitation function at low energies. Consequently, its effect cannot be seen clearly in the fusion excitation function and concentrates in relatively high energy region in the fusion barrier distribution. This contrasts with higher order deformations, which affect the fusion excitation fusion at low energies as well, and hence the fusion barrier distribution over all energy region.

\section{EFFECT OF PAIR NEUTRON TRANSFER}

Before we close the paper, we would like to comment on possible effects of pair neutron transfer channel on the fusion reactions. Refs. [29,30] claim that positive Q-value pair neutron transfer channels explain the isotope effects, seen for example in ${ }^{58} \mathrm{Ni}+{ }^{58} \mathrm{Ni},{ }^{58} \mathrm{Ni}+{ }^{64} \mathrm{Ni}$ and ${ }^{64} \mathrm{Ni}+{ }^{64} \mathrm{Ni}$ fusion reactions by enhancing the fusion cross section at low energies in ${ }^{58} \mathrm{Ni}+{ }^{64} \mathrm{Ni}$ collision. Similarly, by studying ${ }^{28} \mathrm{Si}+{ }^{68} \mathrm{Zn}$ scattering at sub-barrier energies, Ref. [31 claims that the coupling of the positive Q-value two neutron transfer channel significantly enhances the fusion cross section.

Among the three reactions which we discuss in this paper, only the ${ }^{16} \mathrm{O}+{ }^{238} \mathrm{U}$ has a two neutron transfer channel whose $\mathrm{Q}$-value is positive, the $\mathrm{Q}$-value for the two neutron pick-up reaction from ${ }^{16} \mathrm{O}+{ }^{238} \mathrm{U}$ to ${ }^{18} \mathrm{O}+{ }^{236} \mathrm{U}$ being $0.826 \mathrm{MeV}$ in the ground state channel. This transfer channel might resolve the discrepancy between the experimental data and the coupled-channels calculations in the fusion excitation function at low energies for the ${ }^{16} \mathrm{O}+{ }^{238} \mathrm{U}$ fusion reaction (see Fig.6). In order to see this possibility, we study here the effects of this transfer channel following the prescription in Ref. [29], where the transfer reaction is treated in the same way as a vibrational excitation in the coupled-channels formalism. The form factor of the transfer reaction is assumed to be

$$
F_{\text {trans }}(R, \theta)=-\sigma_{t} \frac{d V_{N}(R, \theta)}{d R},
$$

where $\sigma_{t}$ is the strength parameter of the transfer reaction, $V_{N}(R, \theta)$ is the deformed ion-ion potential. This form factor is slightly simplified from that in Refs. [32,33] by ignoring a small correction term. We determine the strength parameter by fitting the excitation function of the fusion cross sections. The optimum set of deformation parameters are readjusted by the $\chi^{2}$ fitting to the experimental data after including pair neutron transfer.

The results are shown in Fig.7. The solid line includes the effect of the transfer reaction, while the dashed line takes only the rotational excitation into account. We left out the effects of vibrational coupling in these calculations. We see that the transfer channel significantly enhances the fusion cross section at low energies. The optimum deformation parameters in this analysis are $\beta_{2}=0.299, \beta_{4}=0.002$ and $\beta_{6}=0.034$. Unfortunately, the dimension of the coupled-channels calculations becomes too large to simultaneously take both effects of transfer reaction and vibrational excitations into account. In the summary section, we also mention the possible importance of single nucleon transfer reactions.

\section{SUMMARY}

We studied the effects of $\beta_{6}$ deformation on heavy ion fusion reactions at energies near and below the Coulomb barrier by analyzing the excitation function of the fusion cross section and fusion barrier distribution for ${ }^{16} \mathrm{O}+{ }^{154} \mathrm{Sm}$, ${ }^{186} \mathrm{~W}$ and ${ }^{238} \mathrm{U}$ reactions. Coupled-channels equations have been solved by taking the rotational excitation, and the octupole, $\beta$ and $\gamma$ vibrations of the target nuclei into account stepwise. The calculations which took only the ground state rotational band into account showed that $\beta_{6}$ deformation is important for all three reactions. The optimum value of $\beta_{6}$ well agrees with that obtained from the ground state mass calculations and inelastic neutron scattering for ${ }^{186} \mathrm{~W}$. On the other hand, the sign of $\beta_{6}$ is inconsistent with that from the analyses of inelastic alpha and proton 
scatterings and the ground state mass calculations for ${ }^{154} \mathrm{Sm}$ and ${ }^{238} \mathrm{U}$ targets. We examined the validity of the linear approximation we took for the Coulomb coupling and gave a reasoning to conjecture that it is good enough to determine the optimum values of higher order deformation, i.e. $\beta_{4}$ and $\beta_{6}$, parameters.

We have then shown that the coupling to the low-lying octupole vibration significantly affects the fusion barrier distribution in the ${ }^{16} \mathrm{O}+{ }^{154} \mathrm{Sm},{ }^{238} \mathrm{U}$ reactions. Interestingly, it changed the sign of the optimum $\beta_{6}$ to agree with that suggested from non-fusion analyses. The $\beta$ and $\gamma$ vibrations are then also taken into account. We found that the $\beta$ vibration introduces only negligible effect, while the $\gamma$ vibration changes the fusion barrier distribution by a noticeable amount, though the change is less than that due to higher order, i.e. $\beta_{4}$ and $\beta_{6}$, deformations. An interesting feature is that the $\gamma$ band does not affect the fusion excitation function at low energies, and hence its effect mostly appears in the fusion barrier distribution in relatively high energy region. This contrasts with higher order deformations, which influence the fusion cross section at low energies as well, and hence the fusion barrier distribution over all energy region. We left $\chi^{2}$ fitting to optimize the deformation parameters to a future work because of the computational heaviness.

A problem with the ${ }^{16} \mathrm{O}+{ }^{238} \mathrm{U}$ fusion reaction is that the coupled-channels calculations which include only rotational and vibrational excitations cannot reproduce large experimental fusion cross section at low energies. We showed in sect.VII that two neutron transfer reaction enhances the fusion cross section at low energies. One will, however, need to study the effects of single nucleon transfer reactions as well in order to reach a comprehensive understanding of this reaction and to draw a conclusive conclusion. In this connection, it is interesting to notice that larger experimental cross sections for one nucleon transfer reactions than those for two nucleon transfer reactions at low energies have been reported for several systems 34 36].

Finally, we wish to make some comments on the limitations of our theoretical framework. We assumed a simple Coulomb interaction given by Eq. (A4), which has a few shortcomings. The first is that the bare Coulomb interaction is identified with the Coulomb interaction between two point charges instead of the Coulomb potential for a uniformly charged extended object, which is often used for heavy-ion collisions. The second is that the same Coulomb coupling form factor, which is valid only in the region where there is no overlap between the projectile and target nuclei, is used over all separation distance. Furthermore, the Coulomb and the nuclear deformation parameters are assumed to be the same. The first two problems are related to each other, and might cause non-negligible effects, since the turning point at the inner side of the potential barrier is located sometimes inside the standard choice of the so called Coulomb radius, $R_{C}=1.2\left(A_{P}^{1 / 3}+A_{T}^{1 / 3}\right) \mathrm{fm}$, or even the sum of the radii of the projectile and target nuclei. The analyses which allow different values for the Coulomb and the nuclear deformation parameters carry one of the important advantages of heavy-ion fusion reactions compared with the other analyses, say neutron scattering. Such analyses will explore the difference between the charge and matter distributions, and will be very interesting also in connection with the study of the structure of exotic unstable nuclei, which is one of the current interests of nuclear physics. We will discuss in detail the effects of the improvements of theoretical analyses in these three respects in a separate paper [40]. Here we simply wish to mention that the first effect, i.e. the difference of the Coulomb interaction between two point charges from the Coulomb potential for a uniform charge distribution, can be mimiced to a large extent by renormalizing the bare potential.

\section{ACKNOWLEDGMENTS}

We thank D.M. Brink, A.B. Balantekin, A. Ono, M. Ueda, N. Ihara and K. Yoshizaki for useful discussions. This research was supported by the Monbusho Scholarship and the International Scientific Research Program: Joint Research: contract number 09044051 from the Japanese Ministry of Education, Science and Culture.

\section{APPENDIX A: BRIEF DESCRIPTION OF THE COUPLED-CHANNELS FORMALISM}

In this appendix we briefly explain the coupled-channels formalism which we used. We present here the case, where all the $\beta, \gamma$ and octupole vibrations are taken into account. The total Hamiltonian reads

$$
H=T+H_{\text {int }}(\xi)+V(\boldsymbol{R}, \xi),
$$

where $T$ is the kinetic energy of the relative motion between the projectile and target, $H_{\text {int }}(\xi)$ the Hamiltonian of the intrinsic motions of the colliding nuclei, whose coordinates are denoted by $\xi$, and $V(\boldsymbol{R}, \xi)$ the interaction Hamiltonian which depends on the coordinates of both the relative motion, $\mathbf{R}$, and nuclear intrinsic motions.

We use the geometrical collective model for nuclear intrinsic motions. The variables $\xi$ are then the static as well as dynamic deformation parameters specifying the radius of the target nucleus as, 


$$
R(\theta, \phi, a)=R_{T}\left[1+\sum_{\lambda} \beta_{\lambda} Y_{\lambda 0}(\theta)+a_{20}^{\prime} Y_{20}(\theta)+a_{22}^{\prime}\left[Y_{22}(\theta, \phi)+Y_{2-2}(\theta, \phi)\right]+a_{30} Y_{30}(\theta)\right] .
$$

In writing Eq. A2 we chose the rotating coordinate frame, where the z-axis is taken to be parallel to the coordinate of the relative motion $\mathbf{R}[23]$. $\theta$ and $\phi$ are Euler angles which define the orientation of the principal axes of the deformed target in this frame. $\beta_{\lambda}, \lambda$ being 2,4 and 6 , are the static deformation parameters. $a$ means $a_{20}^{\prime}, a_{22}^{\prime}$ and $a_{30}$ which are the dynamical deformation parameters describing the $\beta, \gamma$ and octupole $\mathrm{K}=0^{-}$vibrations, respectively.

The interaction Hamiltonian $V(\boldsymbol{R}, \xi)$ consists of the nuclear and Coulomb parts. We assume the former to be

$$
V_{N}(R, \theta, \phi, a)=\frac{-V_{0}}{1+\exp \left[\left(R-R_{P}-R(\theta, \phi, a)\right) / a_{0}\right]} .
$$

It contains both the bare potential and the coupling Hamiltonian. When we treat the channel-coupling in the perturbation theory, say, of first or second order, we expand $V_{N}(R, \theta, \phi, a)$ with the relevant deformation parameters. The actual procedure of the full order coupled-channels calculations is explained in Refs. [37, 38].

We assume a simple Coulomb interaction by ignoring the change of the analytic expressions of the bare Coulomb interaction and the Coulomb coupling form factor depending on the relative magnitude between the distance $\mathrm{R}$ and either the sum of the charge radii of the projectile and target or the absolute value of their difference. The formula we take reads up to the leading order of the dynamical variables as

$$
\begin{aligned}
V_{C}(R, \theta, \phi, a)= & \frac{Z_{P} Z_{T} e^{2}}{R}+\sum_{\lambda} \frac{3 Z_{P} Z_{T} e^{2}}{2 \lambda+1} \frac{R_{T}^{\lambda}}{R^{\lambda+1}}\left[\beta_{\lambda} Y_{\lambda 0}(\theta)+a_{\lambda 0}^{\prime} Y_{\lambda 0}(\theta) \delta_{\lambda, 2}\right. \\
& \left.+\delta_{\lambda, 2} a_{\lambda 2}^{\prime}\left(Y_{\lambda 2}(\theta, \phi)+Y_{\lambda-2}(\theta, \phi)\right)\right]+\frac{3 Z_{P} Z_{T} e^{2}}{7} a_{30} Y_{30}(\theta) \frac{R_{T}^{3}}{R^{4}} .
\end{aligned}
$$

We assume the same charge radius and deformation parameters as those for the nuclear part for the target nucleus.

The Hamiltonian for the intrinsic motions consists of four parts,

$$
H_{\text {int }}(\xi)=H_{\text {rot }}+H_{\beta}+H_{\gamma}+H_{o} .
$$

They describe the rotational and $\beta, \gamma$ and octupole vibrational excitations. Their explicit forms and the corresponding eigenfunctions and eigenvalues can be found in Ref. [39]

We introduce two basic approximations. The one is the no-Coriolis approximation and the other the sudden tunneling approximation, i.e. degenerate spectrum approximation, for the rotational motion. The latter corresponds to setting $H_{\text {rot }}$ to be zero. In these approximations, coupled-channels equations are solved for each given set of $(J, \theta, \phi), J$ being the initial angular momentum of the relative motion, by expanding the wave function as

$$
\Psi_{J \theta \phi}\left(R, \xi_{v}\right)=\sum_{n} \frac{\chi_{n}^{J \theta \phi}(R)}{R} \Phi_{n}\left(\xi_{v}\right),
$$

where $\xi_{v}$ represent the coordinates of the $\beta, \gamma$ and octupole vibrations, and $n$ is the abbreviation of a set of corresponding quantum numbers $\left(n_{\beta}, n_{\gamma}, n_{o}\right)$ [39]. Note that the angular part of the wave function for the relative motion is simply a constant in the rotating frame approximation. We consider only 0 or 1 for all the vibrational quantum numbers. The coupled-channels equations read

$$
\left[-\frac{\hbar^{2}}{2 \mu} \frac{d^{2}}{d R^{2}}+\frac{J(J+1) \hbar^{2}}{2 \mu R^{2}}+\epsilon_{m}+V(R, \theta, a=0)-E\right] \chi_{m}^{J \theta \phi}(R)=-\sum_{n} V_{m n}(R, \theta, \phi) \chi_{n}^{J \theta \phi}(R),
$$

where $\epsilon_{m}$ is the eigenvalue of the vibration excitations corresponding to the eigenstate $\Phi_{m}\left(\xi_{v}\right)$. We represented the total interaction by separating it into the diagonal $V(R, \theta, a=0)$ and the explicit coupling $V_{m n}$ terms with respect to the vibrational excitations. The latter have been evaluated using the wave functions for vibrational motions given in Ref. [39].

We solve the coupled-channels equations by imposing the incoming wave boundary condition at the position of the s-wave potential minimum, and determine the fusion probability by evaluating the incoming flux in each channel at that position. Once the fusion probability is obtained in this way for a given set of $(J, \theta, \phi)$, the total fusion probability for that partial wave is calculated by taking average over the orientation $(\theta, \phi)$ as given by Eq.(1). The fusion cross section is then calculated by the usual partial wave sum. 


\section{APPENDIX B: HIGHER ORDER COULOMB COUPLING}

Here we present the explicit form of the Coulomb coupling up to the $\mathrm{Y}_{6}$ term when the second order coupling terms are included. Only the major terms are explicitly shown for the second order coupling.

$$
\begin{aligned}
V_{C}(R, \theta) & =\frac{Z_{P} Z_{T} e^{2}}{R}+\frac{3}{5}\left(\beta_{2}+\beta_{2}^{2} \frac{2}{7} \sqrt{\frac{5}{\pi}}+\beta_{2} \beta_{4} \frac{4}{7} \sqrt{\frac{9}{\pi}}\right) Z_{P} Z_{T} e^{2} \frac{R_{T}^{2}}{R^{3}} Y_{20}(\theta) \\
& +\frac{3}{9}\left(\beta_{4}+\beta_{2}^{2} \frac{9}{7} \sqrt{\frac{1}{\pi}}+\beta_{2} \beta_{4} \frac{300}{77} \sqrt{\frac{5}{\pi}}\right) Z_{P} Z_{T} e^{2} \frac{R_{T}^{4}}{R^{5}} Y_{40}(\theta) \\
& +\frac{3}{13}\left(\beta_{6}+\beta_{2} \beta_{4} \frac{20}{143} \sqrt{\frac{45 \times 13}{\pi}}\right) Z_{P} Z_{T} e^{2} \frac{R_{T}^{6}}{R^{7}} Y_{60}(\theta)
\end{aligned}
$$

[1] C.Y. Wong, Phys. Rev. Lett. 31, 766(1973).

[2] L.C. Vaz and J.M. Alexander, Phys. Rev.C10, 464(1974).

[3] R.G. Stokstad, Y. Eisen, S. Kaplains, D. Pelte, U. Smilansky, and I. Tserruya, Phys. Rev. Lett.41, 465(1978); Phys. Rev. C21, 2427(1980).

[4] W. Reisdorf et al., Phys. Rev. Lett., 49, 1811(1982).

[5] W. Reisdorf et al., Nucl.Phys., A438, 212(1985).

[6] R.C. Lemmon, J.R. Leigh, J.X. Wei, C.R. Morton, D.J. Hinde, J.O. Newton, J.C. Mein, and M. Dasgupta, Phys. Lett. B316, 32(1993).

[7] J.R. Leigh, M. Dasgupta, D.J. Hinde, J.C. Mein, C.R. Morton, R.C. Lemmon, J.P. Lestone, J.O. Newton, Phys. Rev. C52, 3151 (1995).

[8] Alan H. Shaw and J. S. Greenberg Phys. Rev. C10, 263(1974)

[9] H. Fischer, D. Kamke, H.J.Kittling, E.Kuhlman, H.Plicht, and R. Schormann, Phys. Rev. C15, 921(1977).

[10] J. R. Leigh, N. Rowley, R. C. Lemmon, D. J. Hinde, J. O. Newton, J. X. Wei, J. C. Mein, C. R. Morton, S. Kuyucak and A. T. Kruppa, Phys. Rev. C47, 437(1993)

[11] A. A. Aponik, Jr., C. M. Chesterfield and D. A. Bromley, Nucl. Phys. A159, 367(1970).

[12] G.Palla and H.V.Geramb and C.Pegel Nucl. Phys. A403, 134(1983).

[13] J. P. Delaroche, Phys. Rev. C26, 1899(1982).

[14] D.L. Herdrie, B.G. Harvey, J.R. Meriwether, J. Mahoney, J.c. Fairre and D. G. Kovar, Phys.Rev.Lett.30, 571, (1973).

[15] J.M.Moss, Y.D.Terrien, R.M. Lombard, C. Brassard and J.M. Loiseaux, Phys.Rev.Lett. 26, 1488, (1971).

[16] R.M.Ronningen, R.C.Melin, J.A.Nolen, Jr., and G.M.Crawley and C.E. Bemis, Jr., Phys.Rev.Lett.47, 635, (1981).

[17] L. F. Hansen, I. D. Proctor, D. W. Heikkinen and V.A. Madsen, Phys. Rev. C25, 189(1982).

[18] D. J. Hinde, M. Dasgupta, J. R. Leigh, J. C. Mein, C. R. Morton, J. C. Newton and H. Timmers, Phys. Rev. C53, 1290(1996).

[19] N. Rowley, G.R. Satchler and P.H. Stelson, Phys. Lett. B254, 25(1991).

[20] K. Hagino, N. Takigawa, and A.B. Balantekin, Phys. Rev. C56, 2104 (1997).

[21] K. Hagino, N. Takigawa. M. Dasgupta, D.J. Hinde, and J.R. Leigh, Phys. Rev. Lett. 79, 2014 (1997).

[22] N. Takigawa and K. Ikeda, in Proceedings of the Symposium on The Many Facets of Heavy Ion Fusion Reactions, Argonne National Laboratory Report No. ANL-PHY- 87-1, 1986, p.613.

[23] K. Hagino, N. Takigawa, A.B. Balantekin and J.R. Bennett, Phys. Rev. C52, 286 (1995).

[24] Atomic data and Nuclear data tables P. Möller, J. R. Nix, W. D. Myers and W. J. Swiatecki 59 (1995).

[25] Tamanna Rumin and Noboru Takigawa, unpublished.

[26] R.G.Helmer, Nucl.Data sheets, 52, 1, (1987); Nucl. Phys. A113, 676(1968).

[27] E.N.Shurshikov, Nucl.Data sheets, 53, 601,(1988); Nucl. Phys. A571, 569(1994).

[28] Nucl.Data sheets, 55, 583,(1988); Nucl. Phys. A73, 273(1965); Nucl. Phys. A172, 273(1971); Nucl. Phys. A571, 569(1994).

[29] R.A.Broglia, C.H.Dasso ans S. Landowne, Phys.Rev.32, 1426, (1985).

[30] H.Esbensen and S.Landowne, Nucl.Phys.A492, 473, 1989.

[31] D.O.Kataria, A.K..Sinha, J.J.Das, N.Madhavan, P.Sugathan, Lagy. T.Baby, and I. Mazumdar, R. Singh, C.V.K. baba and Y.K.Agarwal, A.M. Vinodkumar and K.M.Varier, Phys.Rev.56, 1902, (1997).

[32] S. Landowne, C.Price, and H. Esbensen, Nucl.Phys. A484, 98(1988). 
[33] C. H. Dasso, A. Vitturi, Phys. Lett. B179, 337(1986).

[34] R.B. Roberts, S.B. Gazes, J.E. Mason, M. Satteson and S.G.Teichmann, L.L. Lee, Jr.,J.F. Liang J.C. Mahon, and R.J. Vojtech, Phys. Rev. C47, R1831, (1993).

[35] L. Corradi, S. J. Skorka, T. Winkelmann, K. Balog, P. Jänker, H. Leitz, U. Lenz, K.E.G. Löbner, Rudolph, M. Steinmayer, H.G. Thies, B. Million, D.R. Napoli, A.M. Stefanini, S. Beghini, G. Montagnoli, F. Scarlassara, C. Signorini, F. Soramel, Z. Physik A346, 217, (1993).

[36] J.C.Mahon, L.L. Lee Jr., J.F. Liang, C.R. Morton, N.T.P. Bateman, K. Yildiz and B.M. Young, J. Phys. G23,1215, (1997)

[37] K. Hagino, N. Takigawa, M. Dasgupta, D.J. Hinde and J.R. Leigh, Phys. Rev. C55, 276(1997).

[38] K. Hagino, N. Rowley, and A.T. Kruppa, Comp. Phys. Comm., in press.

[39] J.M.Eisenberg and W.Greiner, Nuclear Models, Volume 1, 363, 1987.

[40] N.Takigawa, Tamanna Rumin and N. Ihara, to be published 


\section{Figure Captions}

Fig.1

Comparison of theoretical (a) excitation functions of the fusion cross section and (b) fusion barrier distributions with experimental data [7, 18]. Only ground state rotational excitations are taken into account in the theoretical calculations. The dashed lines represent the optimum fits when only quadrupole deformation is included. The dotted curves include hexadecapole deformation, while the solid lines show the final fits including hexacontatetrapole deformation.

\section{Fig.2}

Sensitivity of (a) the excitation function of the fusion cross section, and (b) the fusion barrier distribution to the hexacontatetrapole deformation. The solid lines were obtained by using the optimum $\beta_{6}$ parameter. The dotted lines were obtained by inverting the sign of $\beta_{6}$, while the dashed lines represent the results when the hexacontatetrapole deformation is set equal to zero.

\section{Fig.3}

Comparison of theoretical (a) excitation functions and (b) fusion barrier distributions calculated by using deformation paramaters from different analyses. The data are taken from Refs. [7].18]. The results using the optimum deformation parameters from the analysis of the fusion data are shown by the solid lines. The dashed lines use the deformation parameters obtained from inelastic scattering [11,13, 17], while the dotted curves those from the nuclear ground state mass calculations 24. All the calculations include hexacontatetrapole deformation.

\section{Fig.4}

Study of the relative importance between the nuclear and the Coulomb $Y_{4}$ couplings. The solid line has been calculated by including both nuclear and Coulomb $\mathrm{Y}_{4}$ couplings, while the dashed line by ignoring the nuclear $\mathrm{Y}_{4}$ coupling. The results, where only the Coulomb $\mathrm{Y}_{4}$ coupling and both the nuclear and Coulomb $\mathrm{Y}_{4}$ cuplings have been discarded are almost the same as the solid and the dashed lines, respectively.

\section{Fig.5}

Effects of octupole vibration. The dashed lines represent the optimum fits when only the ground state rotation band is taken into account, while the solid lines show the optimum fits when the coupling to the $\mathrm{K}=0^{-}$octupole vibration is added.

\section{Fig 6}

The same as Fig.1, but when octupole, $\beta$ and $\gamma$ vibrations are taken into account. The solid line for the ${ }^{186} \mathrm{~W}$ target has been obtained by including only the ground state rotational band, while that for the ${ }^{154} \mathrm{Sm}$ and ${ }^{238} \mathrm{U}$ targets by adding the octupole vibration. The change due to the beta band is invisible. The dotted line has been obtained by adding the $\gamma$ band.

\section{Fig 7}

Effect of pair neutron transfer reaction on the ${ }^{16} \mathrm{O}+{ }^{238} \mathrm{U}$ fusion reactions. The dashed line takes only the ground state rotational excitation into account, while the solid line the pair neutron transfer reaction in addition. 

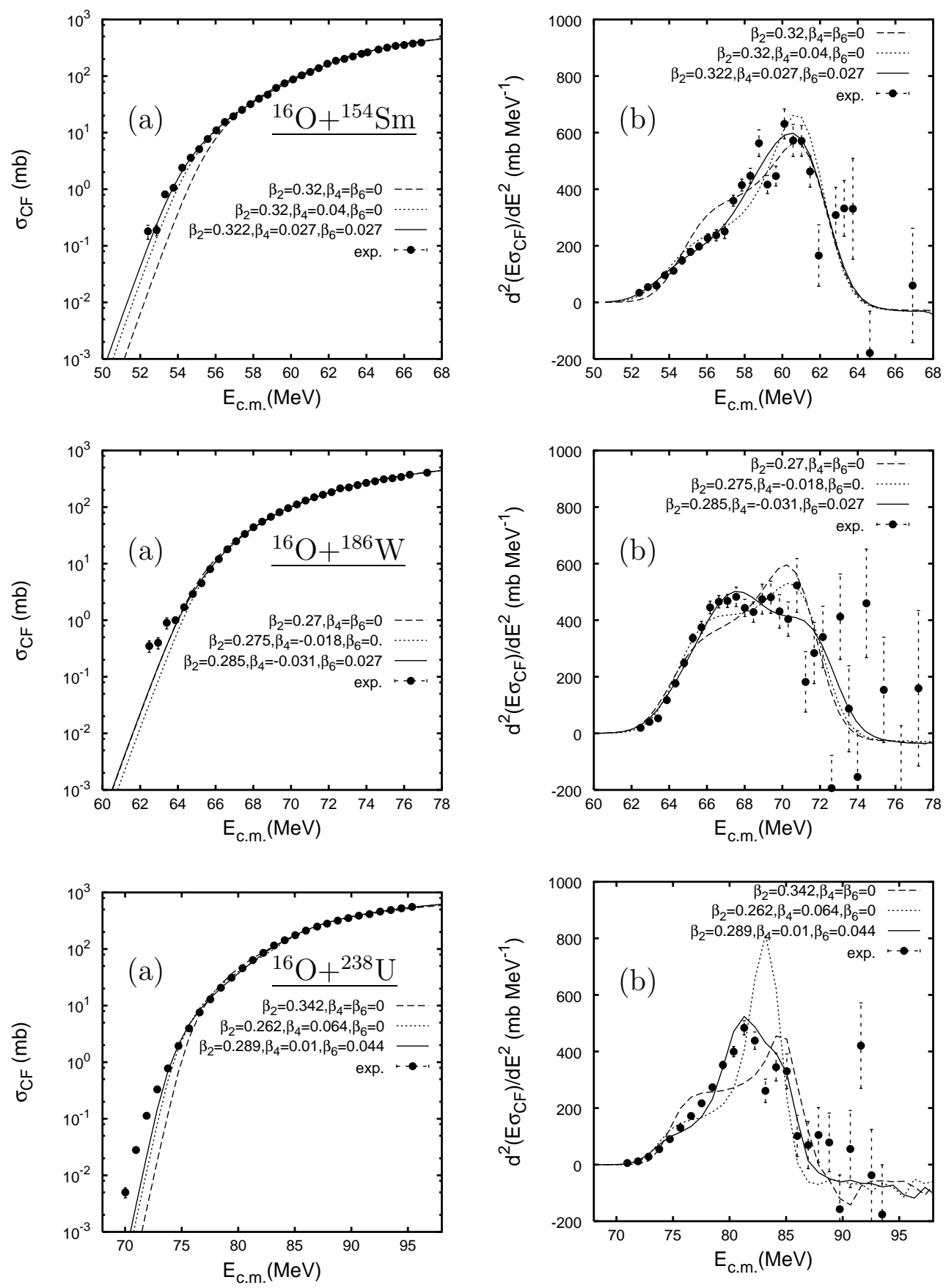

Fig. 1 

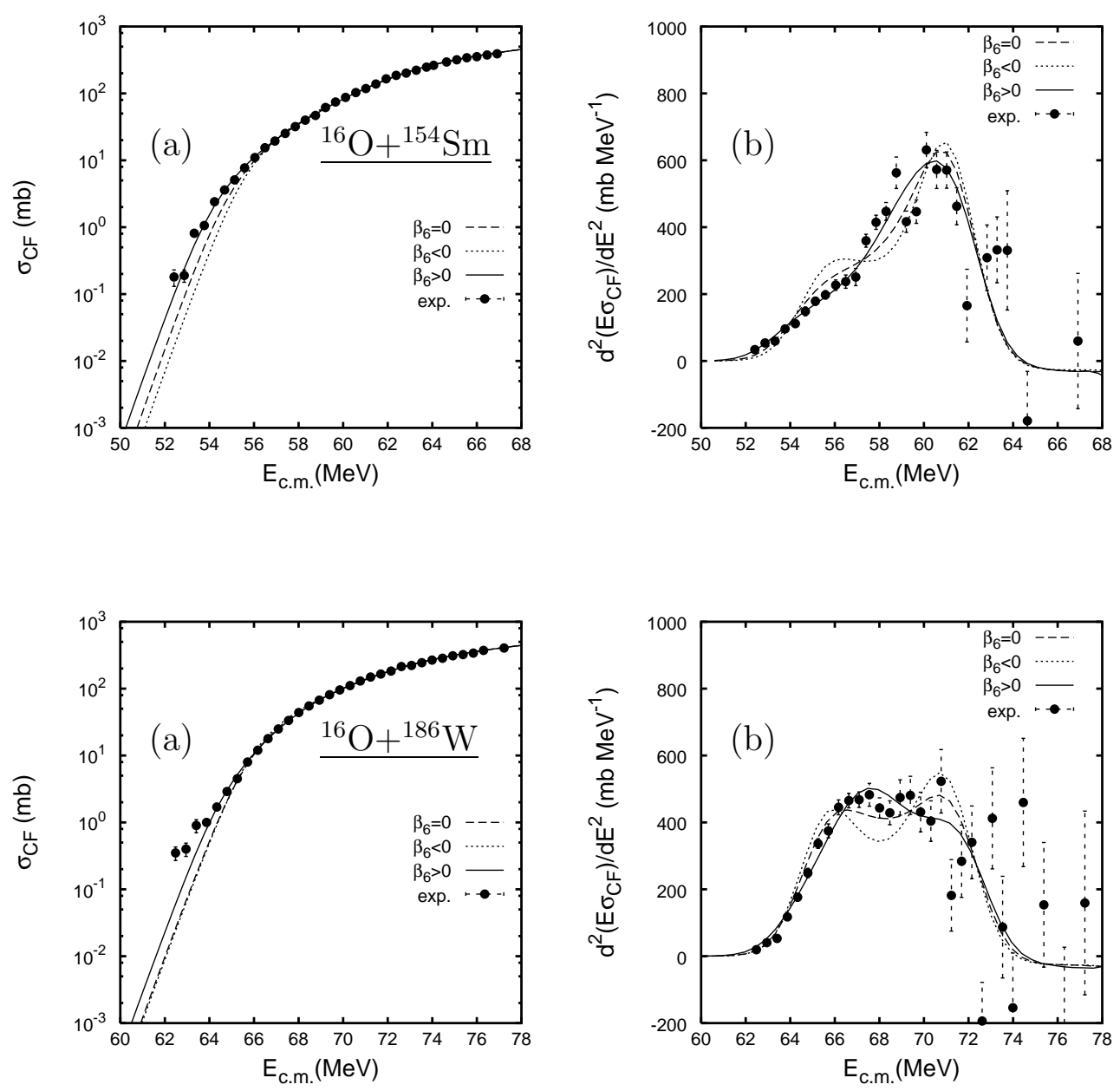

Fig. 2 

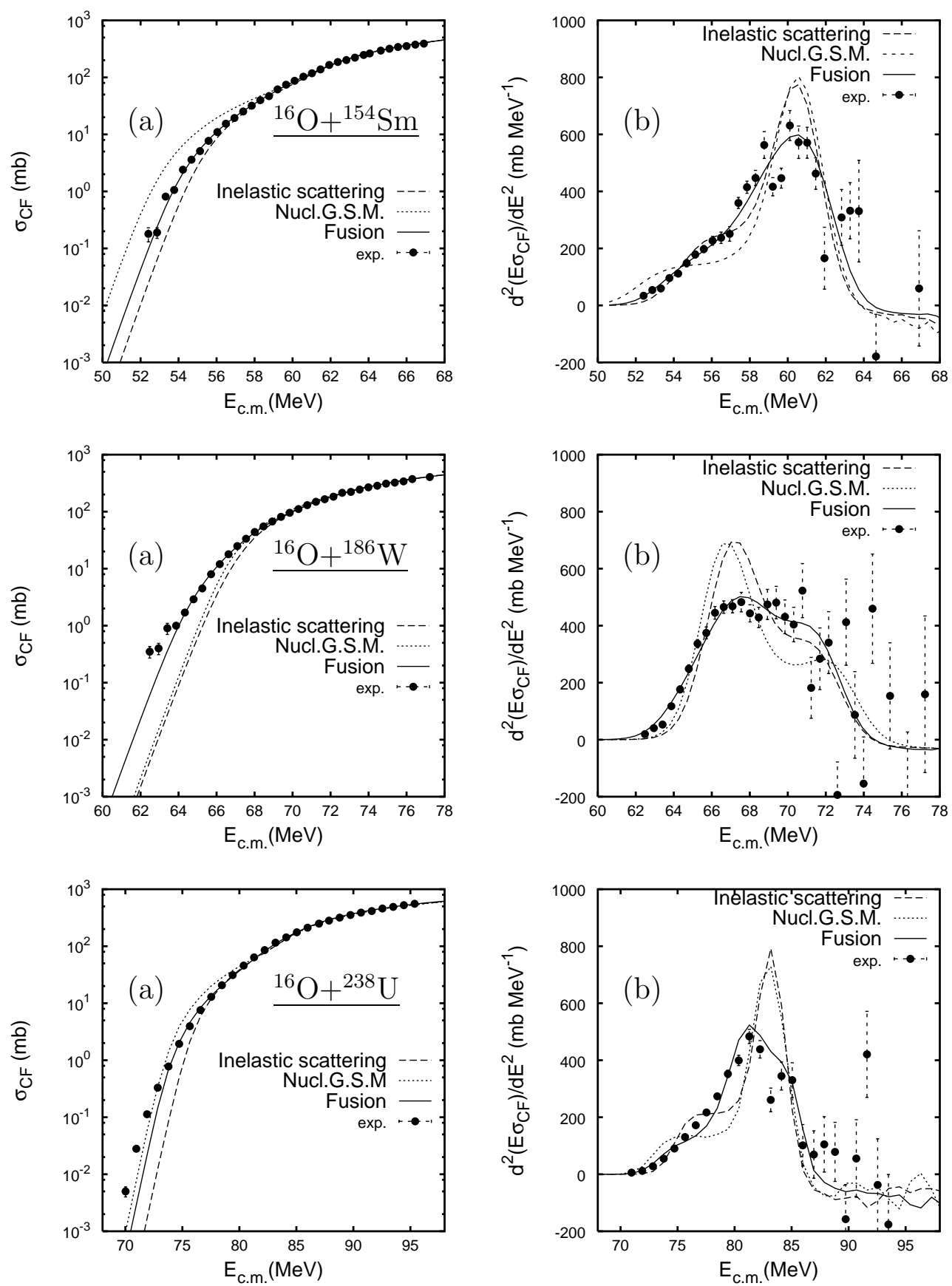

Fig. 3 


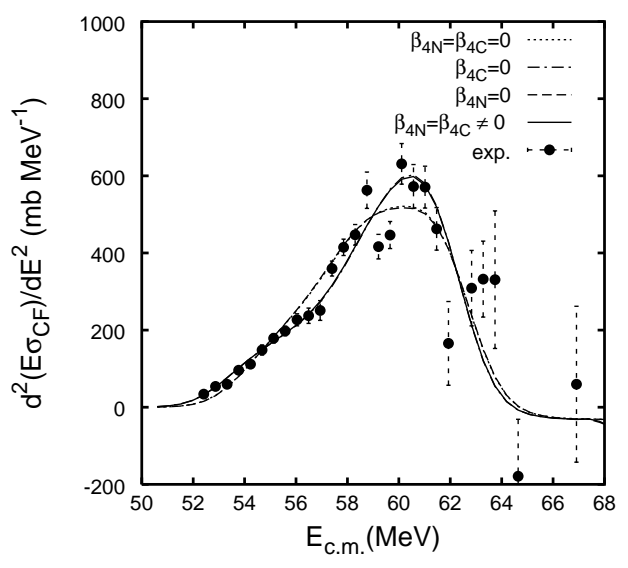

Fig. 4 

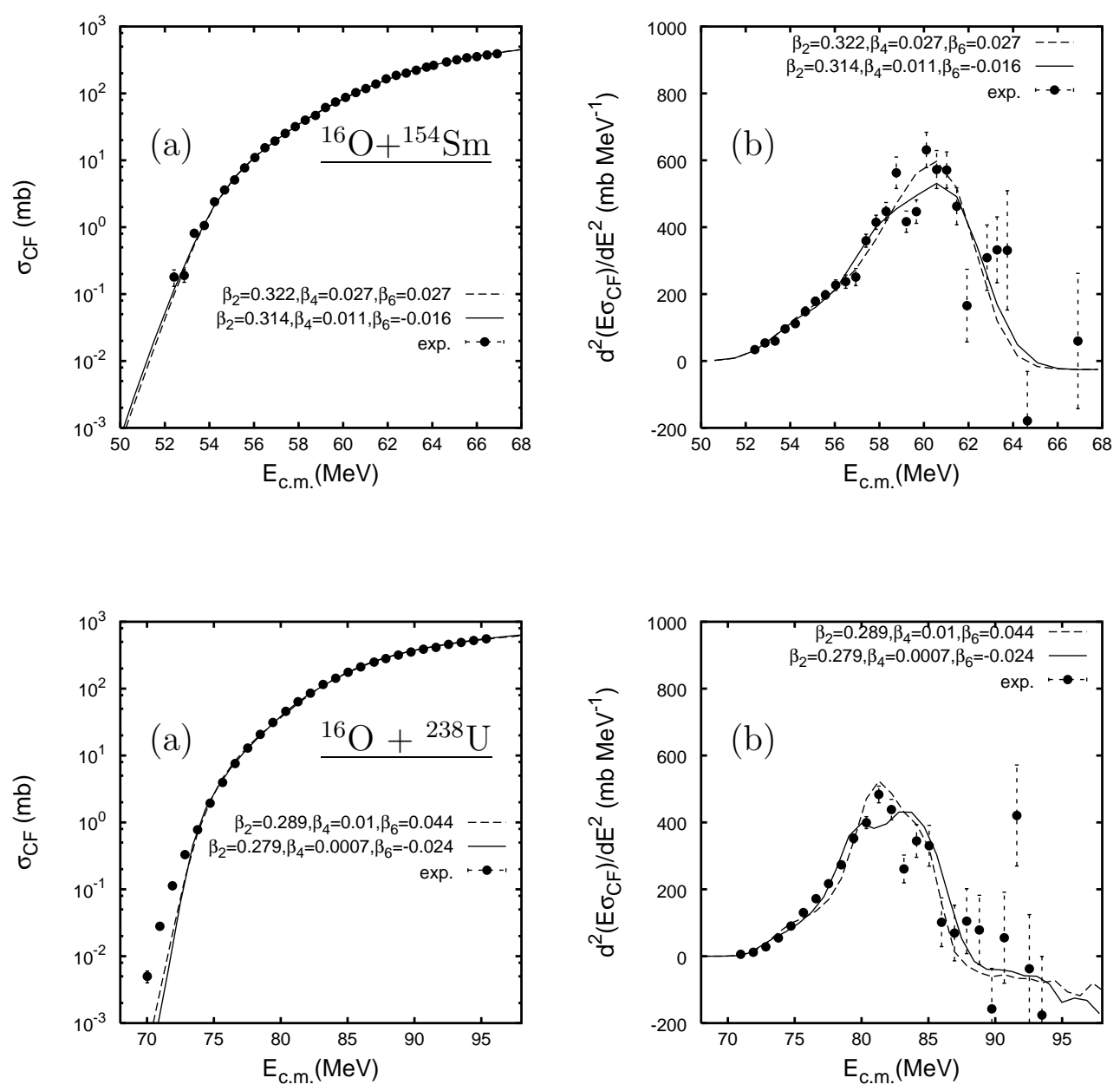

Fig. 5 

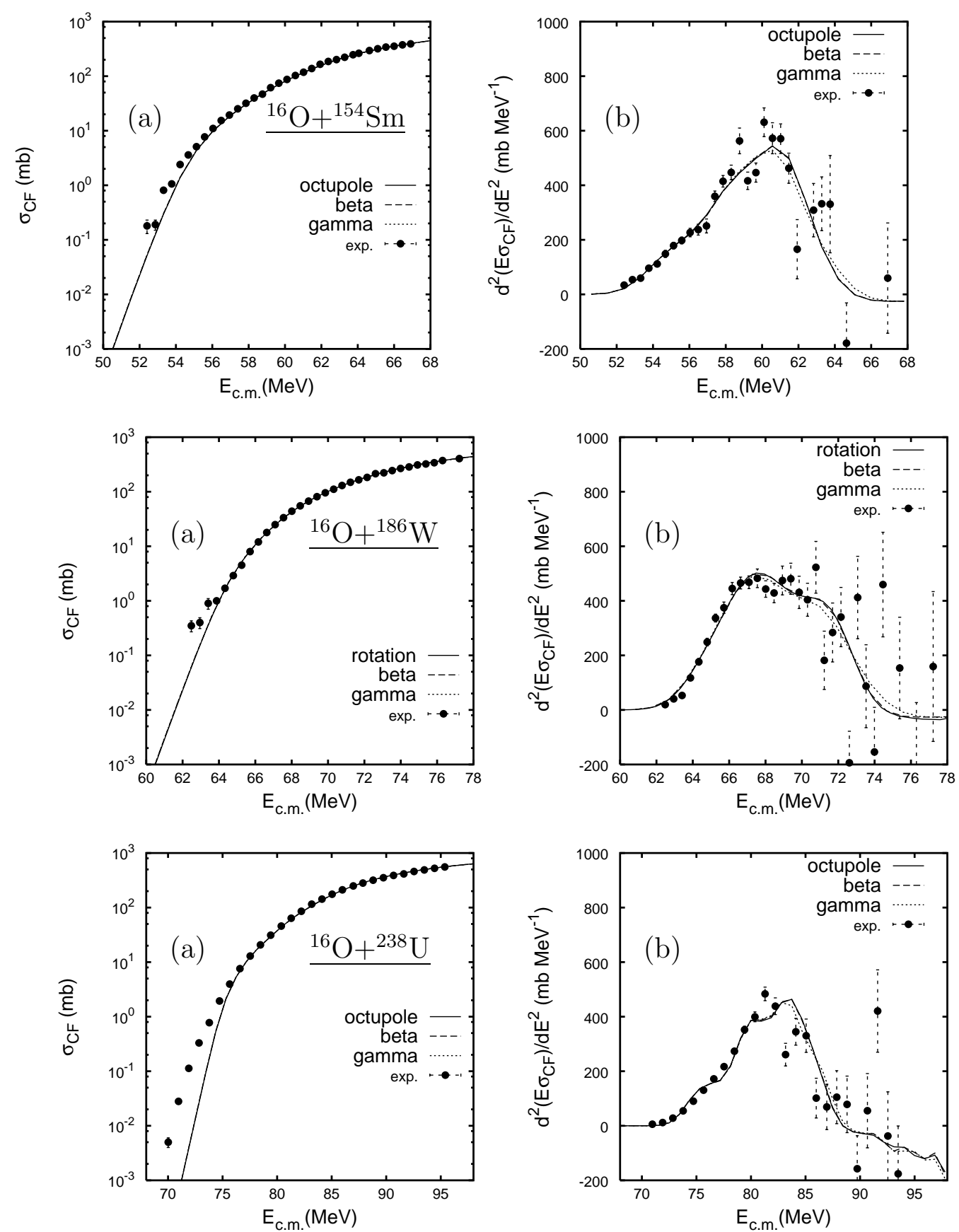

Fig. 6 

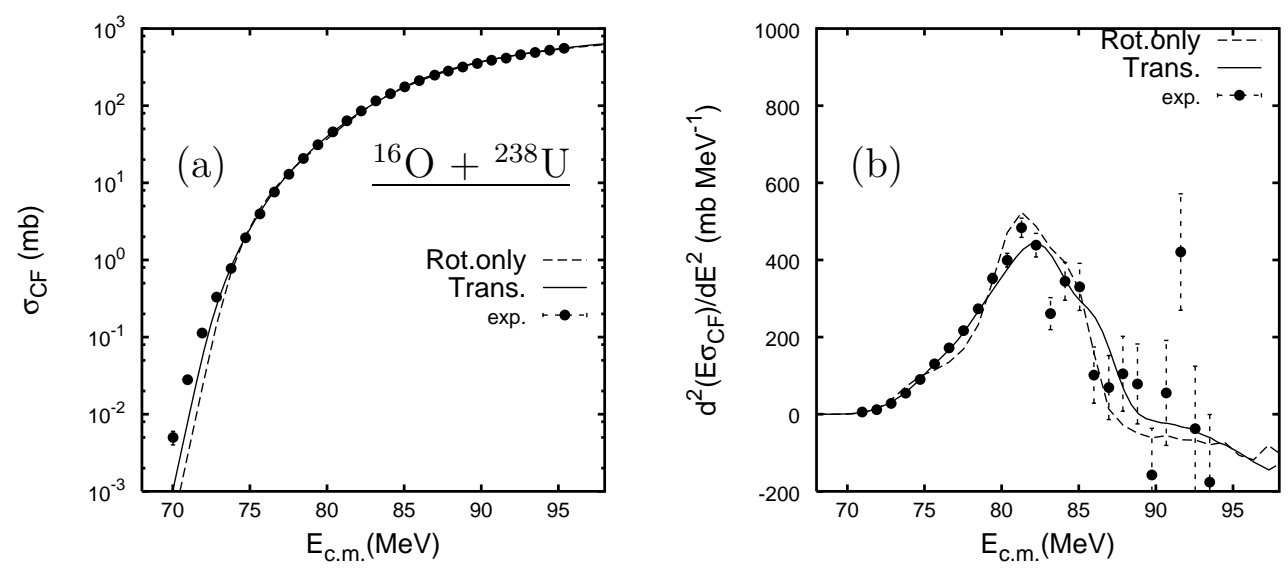

Fig. 7 